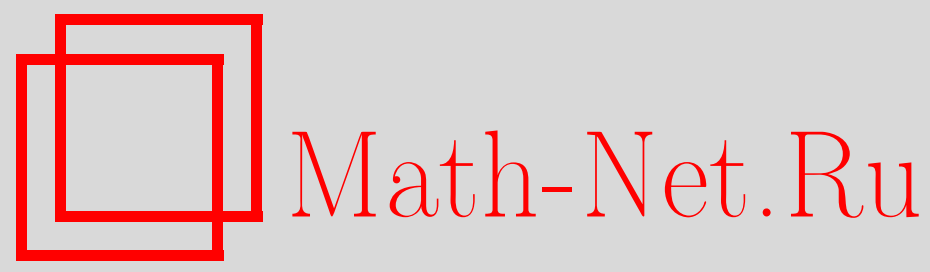

Э. А. Тур, Асимптотика собственных значений для одного класса матриц Якоби с предельным точечным спектром, Матем. заметки, 2003, том 74, выпуск 3, 449-462

DOI: https://doi.org/10.4213/mzm279

Использование Общероссийского математического портала Math-Net.Ru подразумевает, что вы прочитали и согласны с пользовательским соглашением http://www.mathnet.ru/rus/agreement

Параметры загрузки:

IP: 44.207 .124 .84

26 апреля 2023 г., $17: 15: 35$

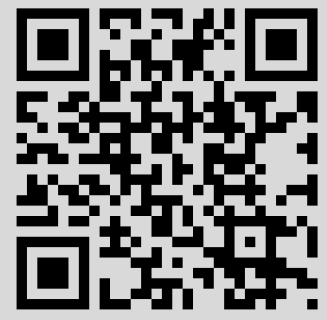




\section{АСИМПТОТИКА СОБСТВЕННЫХ ЗНАЧЕНИЙ ДЛЯ ОДНОГО КЛАССА МАТРИЦ ЯКОБИ С ПРЕДЕЛЬНЫМ ТОЧЕЧНЫМ СПЕКТРОМ}

\section{Э. A. Тyp}

В статье рассмотрен класс матриц Якоби с очень быстро убывающими весами. Показано, что функция Вейля (матричньй элемент резольвенты оператора) для рассматриваемого класса представима в виде отношения двух целых трансцендентных функций нулевого порядка. При этом коэффициенты разложения этих функций в ряд Тейлора пропорциональны производящим функциям числа решений в целых числах определенных диофантовых уравнений. Найдена асимтотическая оценка собственных значений.

Библиография: 8 названий.

Введение. В статье рассматривается класс матриц Якоби вида

$$
A=\left(\begin{array}{ccccc}
0 & b_{0} & 0 & 0 & \ldots \\
b_{0} & 0 & b_{1} & 0 & \ldots \\
0 & b_{1} & 0 & b_{2} & \ldots \\
0 & 0 & b_{2} & 0 & \ldots \\
\ldots & \ldots & \ldots & \ldots & \ldots
\end{array}\right), \quad b_{n-1}=q^{n^{s}}, \quad 0<q<1, \quad s=1,2, \ldots
$$

с нулевой главной диагональю и весами $b_{n}$. Якобиева матрица с такими элементами определяет ограниченный самосопряженньй оператор $A$, действующий в пространстве последовательностей $l_{2}$. Так как $\lim _{n \rightarrow \infty} b_{n}=0$, по теореме М. Г. Крейна [1] оператор $A$ является вполне непрерьвньм и, следовательно, его спектр имеет единственную предельную точку $\lambda=0$. Спектр оператора $A$ полностью определяется функцией $\Gamma$. Вейля $R(\lambda)$ :

$$
R(\lambda)=\left(e_{0}, R_{\lambda} e_{0}\right)=\left(e_{0},(A-\lambda E)^{-1} e_{0}\right),
$$

где $R_{\lambda}$ - резольвента оператора $A$, а $e_{0}$ - первьй вектор базиса, в котором оператор $A$ представлен якобиевой матрищей.

В работе показано, что для указанного выше класса матриц функция $R(\lambda)$ представима в виде отношения двух целых трансцендентных функций переменной $\lambda^{-2}$, т.е.

$$
R(\lambda)=-\frac{f_{1}\left(\lambda^{-2}\right)}{\lambda f_{2}\left(\lambda^{-2}\right)},
$$

где $f_{1}(x)$ и $f_{2}(x)$ - целые функции нулевого порядка (первого порядка и минимального типа). 
Таким образом, исследование поведения собственных значений $\pm \lambda_{n}\left(\lambda_{n}>0, n=\right.$ $0,1,2, \ldots$ ) оператора $A$ сводится к изучению нулей функции $f_{2}(z)$, что и сделано в настоящей работе. Результат можно представить в виде следующей асимптотической оценки:

$$
b_{2 n+1}<\lambda_{n}<b_{2 n-1}, \quad n>N
$$

где $N$ - достаточно большое целое число.

1. Функция Вейля как предел отношения двух полиномов. Рассмотрим функцию Вейля $R(\lambda)$ для якобиевой матрицы общего вида

$$
A=\left(\begin{array}{ccccc}
a_{0} & g b_{0} & 0 & 0 & \ldots \\
g b_{0} & a_{1} & g b_{1} & 0 & \ldots \\
0 & g b_{1} & a_{2} & g b_{2} & \ldots \\
0 & 0 & g b_{2} & a_{3} & \ldots \\
\ldots & \ldots & \cdots & \cdots & \ldots
\end{array}\right)
$$

где перед коэффициентами $b_{n}$ введен множителем параметр $g$.

Используем для функции $R(\lambda)$ формулу Стоуна [2]. Эта формула дает представление $R(\lambda)$ в виде непрерывной дроби вида

$$
R(\lambda)=\frac{1}{a_{0}-\lambda-\frac{g^{2} b_{0}^{2}}{a_{1}-\lambda-\frac{g^{2} b_{1}^{2}}{a_{2}-\lambda-\frac{g^{2} b_{2}^{2}}{a_{3}-\lambda-\cdots}}} .}
$$

Обозначая $z=g^{2}$, преобразуем эту дробь к виду

$$
R(\lambda)=\frac{c_{0}(\lambda)}{1+\frac{c_{1}(\lambda) z}{1+\frac{c_{2}(\lambda) z}{1+\cdots}}}
$$

где последовательность функций $c_{n}(\lambda)$ определяется формулами

$$
\begin{aligned}
c_{0}(\lambda) & =\frac{1}{a_{0}-\lambda}, \\
c_{n}(\lambda) & =\frac{-b_{n-1}^{2}}{\left(a_{n-1}-\lambda\right)\left(a_{n}-\lambda\right)}, \quad n=1,2, \ldots
\end{aligned}
$$

В дальнейшем для сокращения будем опускать у функций $c_{n}(\lambda)$ аргумент $\lambda$.

Рассмотрим $n$-ю подходящую дробь к непрерывной дроби (1.1)

$$
R_{n}(\lambda)=\frac{c_{0}}{1+\frac{c_{1} z}{1+\cdots+\frac{c_{n-1} z}{1+c_{n} z}}}=\frac{M_{n}(z)}{N_{n}(z)} .
$$


Здесь $M_{n}$ и $N_{n}$ - подходящие числители и знаменатели непрерьвной дроби (1.1), удовлетворяющие, как известно, единым трехчленным рекуррентным соотношениям

$$
Q_{n+2}=Q_{n+1}+c_{n+2} z Q_{n}, \quad n=0,1, \ldots,
$$

с начальньми условиями

$$
M_{0}=c_{0}, \quad M_{1}=c_{0}, \quad N_{0}=1, \quad N_{1}=1+c_{1} z .
$$

Величины $M_{n}(z)$ и $N_{n}(z)$ являются полиномами по $z$. Если $n$ нечетное, то степень $M_{2 k-1}(z)$ равна $(k-1)$, а степень $N_{2 k-1}(z)$ равна $k$. Если $n$ четное, то степени $M_{2 k}(z)$ и $N_{2 k}(z)$ одинаковы и равны $k$. Полиномы $M_{n}(z)$ и $N_{n}(z)$ лиш множителем отличаются от ортогональных полиномов первого и второго рода [3], через отношение которых также может быть выражена подходящая дробь $R_{n}(\lambda)$. Однако их структура намного проще, чем структура ортогональных полиномов. Этим мы и воспользуемся для нахождения явных выражений для коэффициентов при степенях $z$ полиномов $M_{n}(z)$ и $N_{n}(z)$. Так как вькладки для полиномов $M_{n}(z)$ и $N_{n}(z)$ идентичны, проведем их подробно только для полиномов $N_{n}(z)$. Из сказанного вьше следует, что эти полиномы можно представить в виде

$$
N_{n}(z)=\sum_{m=0}^{N} B_{m}^{(n)} z^{m}, \quad \begin{cases}N=n / 2, & n \equiv 0(\bmod 2), \\ N=(n+1) / 2, & n \equiv 1(\bmod 2) .\end{cases}
$$

Подставляя это выражение в рекуррентные соотношения (1.3) и учитьвая начальные условия (1.4), нетрудно получить следующие рекуррентные соотношения для коэффициентов $B_{m}^{(n)}$ :

$$
\begin{aligned}
& B_{0}^{(n)}=1, \quad B_{1}^{(n)}=\sum_{k=1}^{n} c_{n}, \quad n \geqslant 1, \\
& B_{2}^{(n)}=c_{3} B_{1}^{(1)}+c_{4} B_{1}^{(2)}+\cdots+c_{n} B_{1}^{(n-2)}, \quad n \geqslant 3, \\
& B_{3}^{(n)}=c_{5} B_{2}^{(3)}+c_{6} B_{2}^{(4)}+\cdots+c_{n} B_{2}^{(n-2)}, \quad n \geqslant 5 \text {, } \\
& \begin{cases}B_{(n-2) / 2}^{(n)}=c_{n-3} B_{(n-4) / 2}^{(n-5)}+\cdots+c_{n} B_{(n-4) / 2}^{(n-2)}, & n \equiv 0(\bmod 2), \\
B_{(n-1) / 2}^{(n)}=c_{n-2} B_{(n-3) / 2}^{(n-4)}+c_{n-1} B_{(n-3) / 2}^{(n-3)}+c_{n} B_{(n-3) / 2}^{(n-2)}, & n \equiv 1(\bmod 2),\end{cases} \\
& \begin{cases}B_{n / 2}^{(n)}=c_{n-1} B_{(n-2) / 2}^{(n-3)}+c_{n} B_{(n-2) / 2}^{(n-2)}, & n \equiv 0(\bmod 2), \\
B_{(n+1) / 2}^{(n)}=c_{n} B_{(n-1) / 2}^{(n-2)}=c_{1} \cdot c_{3} \cdot \ldots \cdot c_{n}, & n \equiv 1(\bmod 2) .\end{cases}
\end{aligned}
$$

Из этих соотношений нетрудно получить компактноевыражение коэффициентов $B_{m}^{(n)}$ через функции $c_{n}$ :

$$
B_{m}^{(n)}=\sum_{n_{1}=2 m-1}^{n} \sum_{n_{2}=2 m-3}^{n_{1}-2} \cdots \sum_{n_{m}=1}^{n_{m-1}-2} c_{n_{1}} \cdot c_{n_{2}} \cdot \ldots \cdot c_{n_{m}}, \quad B_{0}^{(n)}=1 .
$$


Вводя новые индексы суммирования $n_{1}=s_{1}+2 m-1, n_{2}=s_{2}+2 m-3$ и т.д., можно записать это выражение также в виде

$$
B_{m}^{(n)}=\sum_{s_{1}=0}^{n-2 m+1} \sum_{s_{2}=0}^{s_{1}} \cdots \sum_{s_{m}=0}^{s_{m-1}} c_{s_{1}+2 m-1} \cdot c_{s_{2}+2 m-3} \cdot \ldots \cdot c_{s_{m}+1}, \quad B_{0}^{(n)}=1 .
$$

Точно так же можно получить аналогичную формулу и для коэффищиентов полинома $M_{n}(z)$. Если обозначить

$$
M_{n}(z)=\sum_{m=0}^{N} A_{m}^{(n)} z^{m}, \quad \begin{cases}N=n / 2, & n \equiv 0(\bmod 2) \\ N=(n-1) / 2, & n \equiv 1(\bmod 2)\end{cases}
$$

то для коэффициентов $A_{m}^{(n)}$ справедлива формула

$$
A_{m}^{(n)}=c_{0} \sum_{s_{1}=0}^{n-2 m} \sum_{s_{2}=0}^{s_{1}} \cdots \sum_{s_{m}=0}^{s_{m-1}} c_{s_{1}+2 m} \cdot c_{s_{2}+2 m-2} \cdot \ldots \cdot c_{s_{m}+2}, \quad A_{0}^{(n)}=c_{0} .
$$

Таким образом, мынашли, что подходящая дробь $R_{n}(\lambda)$ к функции Вейля равна отношению двух неортогональных полиномов по параметру $z$ и установили явные выражения для коэффициентов при степенях $z$ этих полиномов. Окончательно можно записать

$$
\begin{aligned}
& R_{n}(\lambda)=\frac{M_{n}(z)}{N_{n}(z)}=\frac{\sum_{m=0}^{N_{1}} A_{m}^{(n)} z^{m}}{\sum_{m=0}^{N_{2}} B_{m}^{(n)} z^{m}},
\end{aligned}
$$

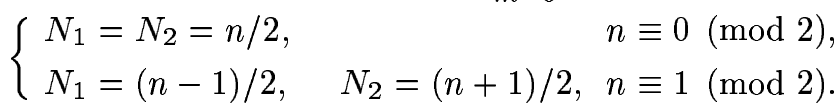

Здесь согласно формулам $(1.2),(1.5),(1.6)$ коэффициенты $A_{m}^{(n)}$ и $B_{m}^{(n)}$ являются функциями $\lambda$.

2. Приложение к рассматриваемому классу весов. Функция Вейля как отношение двух целых трансцендентных функций. Перейдем к частному случаю матриц Якоби, указанному во введении, т.е. рассмотрим веса вида

$$
a_{n}=0, \quad b_{n-1}=q^{n^{s}}, \quad 0<q<1, \quad s=1,2, \ldots .
$$

В этом случае формулы (1.2) предыдушего пункта дают

$$
c_{0}(\lambda)=-\frac{1}{\lambda}, \quad c_{n}(\lambda)=-\frac{b_{n-1}^{2}}{\lambda^{2}}=-\frac{q^{2 n^{s}}}{\lambda^{2}}, \quad n=1,2, \ldots
$$

Следовательно, для коэффициентов $A_{m}^{(n)}$ и $B_{m}^{(n)}$ из формул $(1.5),(1.6)$ получим

$$
\begin{aligned}
B_{m}^{(n)} & =\frac{(-1)^{m}}{\lambda^{2 m}} \\
& \times \sum_{s_{1}=0}^{n-2 m+1} \sum_{s_{2}=0}^{s_{1}} \cdots \sum_{s_{m}=0}^{s_{m-1}} u^{\left(s_{1}+2 m-1\right)^{s}+\left(s_{2}+2 m-3\right)^{s}+\cdots+\left(s_{m}+1\right)^{s}}, \quad u=q^{2},(2.1 \\
A_{m}^{(n)} & =\frac{(-1)^{m+1}}{\lambda^{2 m+1}} \\
& \times \sum_{s_{1}=0}^{n-2 m} \sum_{s_{2}=0}^{s_{1}} \cdots \sum_{s_{m}=0}^{s_{m-1}} u^{\left(s_{1}+2 m\right)^{s}+\left(s_{2}+2 m-2\right)^{s}+\cdots+\left(s_{m}+2\right)^{s}}, \quad u=q^{2} .
\end{aligned}
$$


Введем обозначения

$$
\begin{gathered}
\sigma_{m}\left(s_{1}, s_{2}, \ldots, s_{m}\right)=\left(s_{1}+2 m-1\right)^{s}+\left(s_{2}+2 m-3\right)^{s}+\cdots+\left(s_{m}+1\right)^{s} \\
\pi_{m}\left(s_{1}, s_{2}, \ldots, s_{m}\right)=\left(s_{1}+2 m\right)^{s}+\left(s_{2}+2 m-2\right)^{s}+\cdots+\left(s_{m}+2\right)^{s} \\
\beta_{m}^{(n)}(u)=\sum_{s_{1}=0}^{n-2 m+1} \sum_{s_{2}=0}^{s_{1}} \cdots \sum_{s_{m}=0}^{s_{m-1}} u^{\sigma_{m}\left(s_{1}, s_{2}, \ldots, s_{m}\right)-\sigma_{m}(0,0, \ldots, 0)} \\
\gamma_{m}^{(n)}(u)=\sum_{s_{1}=0}^{n-2 m} \sum_{s_{2}=0}^{s_{1}} \cdots \sum_{s_{m}=0}^{s_{m-1}} u^{\pi_{m}\left(s_{1}, s_{2}, \ldots, s_{m}\right)-\pi_{m}(0,0, \ldots, 0)}
\end{gathered}
$$

Тогда формулы $(2.1),(2.2)$ запишутся в этих обозначениях в виде

$$
\begin{aligned}
& B_{m}^{(n)}=\frac{(-1)^{m}}{\lambda^{2 m}} u^{\sigma_{m}(0,0, \ldots, 0)} \beta_{m}^{(n)}(u), \\
& A_{m}^{(n)}=\frac{(-1)^{m+1}}{\lambda^{2 m+1}} u^{\pi_{m}(0,0, \ldots, 0)} \gamma_{m}^{(n)}(u) .
\end{aligned}
$$

Рассмотрим величины $\beta_{m}^{(n)}(u)$ и $\gamma_{m}^{(n)}(u)$, определенные формулами $(2.5),(2.6)$. Пусть $\mathcal{E}_{m}^{(\sigma)}-$ множество значений, которые принимает величина $\sigma_{m}\left(s_{1}, s_{2}, \ldots, s_{m}\right)-$ $\sigma_{m}(0,0, \ldots, 0)$ при целых $s_{i}$, удовлетворяющих условию $s_{1} \geqslant s_{2} \geqslant \cdots \geqslant s_{m} \geqslant 0$. Очевидно, что множество $\mathcal{E}_{m}^{(\sigma)}$ является подмножеством множества натуральных чисел, совпадающим с ним при $s=1$. Мы можем заменить в формуле (2.5) многократные суммы одной суммой по множеству $\mathcal{E}_{m}^{(\sigma)}$ :

$$
\beta_{m}^{(n)}(u)=\sum_{k \in \mathcal{E}_{m}^{(\sigma)}} p_{\sigma}(n-2 m+1, m, k) u^{k}
$$

Здесь через $p_{\sigma}(n-2 m+1, m, k)$ обозначено число решений в целых неотрищательньх числах $s_{i}$ уравнения

$$
\begin{gathered}
\sigma_{m}\left(s_{1}, s_{2}, \ldots, s_{m}\right)-\sigma_{m}(0,0, \ldots, 0)=k \\
k \in \mathcal{E}_{m}^{(\sigma)}, \quad n-2 m+1 \geqslant s_{1} \geqslant s_{2} \geqslant \cdots \geqslant s_{m} \geqslant 0,
\end{gathered}
$$

которое, учитывая (2.3), можно переписать в виде

$$
\begin{array}{r}
\left(s_{1}+2 m-1\right)^{s}+\left(s_{2}+2 m-3\right)^{s}+\cdots+\left(s_{m}+1\right)^{s} \\
-(2 m-1)^{s}-(2 m-3)^{s}-\cdots-3^{s}-1=k, \\
k \in \mathcal{E}_{m}^{(\sigma)}, \quad n-2 m+1 \geqslant s_{1} \geqslant s_{2} \geqslant \cdots \geqslant s_{m} \geqslant 0 .
\end{array}
$$

Рассмотрим теперь при фиксированном $m$ предел при $n \rightarrow \infty$ величины $\beta_{m}^{(n)}(u)$, определенной суммой (2.9). При этом, очевидно, снимается ограничение $n-2 m+1 \geqslant s_{1}$, и мы получаем

$$
\beta_{m}(u)=\lim _{n \rightarrow \infty} \beta_{m}^{(n)}(u)=\sum_{k \in \mathcal{E}_{m}^{(\sigma)}} p_{\sigma}(m, k) u^{k}
$$


Здесь $p_{\sigma}(m, k)$ есть число решений уравнения (2.10) без ограничительного условия $n-2 m+1 \geqslant s_{1}$, т.е. $p_{\sigma}(m, k)$ есть число решений уравнения

$$
\begin{gathered}
\left(s_{1}+2 m-1\right)^{s}+\left(s_{2}+2 m-3\right)^{s}+\cdots+\left(s_{m}+1\right)^{s} \\
-(2 m-1)^{s}-(2 m-3)^{s}-\cdots-3^{s}-1=k \\
k \in \mathcal{E}_{m}^{(\sigma)}, \quad s_{1} \geqslant s_{2} \geqslant \cdots \geqslant s_{m} \geqslant 0 .
\end{gathered}
$$

Если в сумме (2.9) число слагаемых конечно в силу условия $n-2 m+1 \geqslant s_{1}$, то в сумме (2.11) число слагаемьх уже неограниченно. Ряд (2.11) является сходящимся при $|u|<1$, что вьполняется в данном случае, так как $u=q^{2}, 0<q<1$. Действительно, мы имеем $p_{\sigma}(m, k)<p(k)$ для любых $m, k$ и $s$, где $p(k)$ - число решений в целых неотрицательных числах уравнения

$$
s_{1}+s_{2}+\cdots+s_{k}=k, \quad s_{1} \geqslant s_{2} \geqslant \cdots \geqslant s_{k} \geqslant 0 .
$$

Поэтому при $0<u<1$

$$
\beta_{m}(u)<\sum_{k=0}^{\infty} p(k) u^{k}=\prod_{k=1}^{\infty} \frac{1}{1-u^{k}} .
$$

Последняя же сумма представляет собой известную производящую функцию для функции разбиений $p(k)$ и является сходящейся при $|u|<1$ (см., например, [4]).

Аналогично, функция $\beta_{m}(u)(2.11)$ является производящей функцией соответствующих величин $p_{\sigma}(m, k)$.

Совершенно так же можно рассмотреть и функцию $\gamma_{m}^{(n)}(u)$, определенную формулой (2.6). Приведем результаты:

$$
\gamma_{m}(u)=\lim _{n \rightarrow \infty} \gamma_{m}^{(n)}(u)=\sum_{k \in \mathcal{E}_{m}^{(\pi)}} p_{\pi}(m, k) u^{k}
$$

где $p_{\pi}(m, k)$ есть число решений в целых неотрицательных числах $s_{i}$ уравнения

$$
\pi_{m}\left(s_{1}, s_{2}, \ldots, s_{m}\right)-\pi_{m}(0,0, \ldots, 0)=k, \quad k \in \mathcal{E}_{m}^{(\pi)}, \quad s_{1} \geqslant s_{2} \geqslant \cdots \geqslant s_{m} \geqslant 0
$$

которое, учитывая (2.4), можно переписать в виде

$$
\begin{gathered}
\left(s_{1}+2 m\right)^{s}+\left(s_{2}+2 m-2\right)^{s}+\cdots+\left(s_{m}+2\right)^{s}-(2 m)^{s}-(2 m-2)^{s}-\cdots-2^{s}=k \\
k \in \mathcal{E}_{m}^{(\pi)}, \quad s_{1} \geqslant s_{2} \geqslant \cdots \geqslant s_{m} \geqslant 0 .
\end{gathered}
$$

Функция $\gamma_{m}(u)$ является производящей функцией величин $p_{\pi}(m, k)$, и ряд $(2.13)$ сходится при $|u|<1$.

Для функций $\beta_{m}(u)$ и $\gamma_{m}(u)$ не сушествует явных аналитических выражений, за исключением случая $s=1$. В случае $s=1$ уравнения (2.12) и (2.14) совпадают и принимают простой вид

$$
s_{1}+s_{2}+\cdots+s_{m}=k, \quad k=0,1,2 \ldots, \quad s_{1} \geqslant s_{2} \geqslant \cdots \geqslant s_{m} \geqslant 0 .
$$


Поэтому в этом случае функции $\beta_{m}(u)$ и $\gamma_{m}(u)$ являются производяшими функциями функции разбиений на не более чем $m$ частей и равны [4]

$$
\beta_{m}(u)=\gamma_{m}(u)=\prod_{k=1}^{m} \frac{1}{1-u^{k}}, \quad s=1 \text {. }
$$

В дальнейшем, однако, нам не потребуется точньй вид функций $\beta_{m}(u)$ и $\gamma_{m}(u)$ в случае произвольного $s$. Отметим только очевидные неравенства

$$
\begin{array}{ll}
1<\beta_{m}(u) \leqslant \prod_{k=1}^{m} \frac{1}{1-u^{k}}, \quad \lim _{m \rightarrow \infty} \beta_{m}(u)=\beta(u)<\infty, \\
1<\gamma_{m}(u) \leqslant \prod_{k=1}^{m} \frac{1}{1-u^{k}}, \quad \lim _{m \rightarrow \infty} \gamma_{m}(u)=\gamma(u)<\infty,
\end{array}
$$

Вернемся к полиномам $M_{n}(z)$ и $N_{n}(z)$. Мы имели для них формулы

$$
M_{n}(z)=\sum_{m=0}^{N_{1}} A_{m}^{(n)} z^{m}, \quad N_{n}(z)=\sum_{m=0}^{N_{2}} B_{m}^{(n)} z^{m}
$$

или, учитьвая формулы $(2.7),(2.8)$,

$$
\begin{aligned}
& M_{n}(z)=\sum_{m=0}^{N_{1}} \frac{(-1)^{m+1}}{\lambda^{2 m+1}} u^{\pi_{m}(0,0, \ldots, 0)} \gamma_{m}^{(n)}(u) z^{m} \\
& N_{n}(z)=\sum_{m=0}^{N_{2}} \frac{(-1)^{m}}{\lambda^{2 m}} u^{\sigma_{m}(0,0, \ldots, 0)} \beta_{m}^{(n)}(u) z^{m}
\end{aligned}
$$

Устремляя $n \rightarrow \infty$ и используя полученные результаты, имеем

$$
\begin{aligned}
& M(z)=\lim _{n \rightarrow \infty} M_{n}(z)=\sum_{m=0}^{\infty} \frac{(-1)^{m+1}}{\lambda^{2 m+1}} u^{\pi_{m}} \gamma_{m}(u) z^{m}, \\
& N(z)=\lim _{n \rightarrow \infty} N_{n}(z)=\sum_{m=0}^{\infty} \frac{(-1)^{m}}{\lambda^{2 m}} u^{\sigma_{m}} \beta_{m}(u) z^{m}
\end{aligned}
$$

где мы ввели сокращенные обозначения

$$
\begin{aligned}
& \pi_{m} \equiv \pi_{m}(0, \ldots, 0)=(2 m)^{s}+(2 m-2)^{s}+\cdots+2^{s} \\
& \sigma_{m} \equiv \sigma_{m}(0, \ldots, 0)=(2 m-1)^{s}+(2 m-3)^{s}+\cdots+3^{s}+1 .
\end{aligned}
$$

Оценим величины $\pi_{m}$ и $\sigma_{m}$ при больших $m$. Используя формулу суммирования Эйлера-Маклорена, можно получить следуюшие асимптотические выражения для этих величин:

$$
\pi_{m}=\frac{2^{s}}{s+1} m^{s+1}\left(1+O\left(m^{-1}\right)\right), \quad \sigma_{m}=\frac{2^{s}}{s+1} m^{s+1}\left(1+O\left(m^{-2}\right)\right), \quad m \rightarrow \infty .
$$


Из этих формул следует, что величины $u^{\pi_{m}}$ и $u^{\sigma_{m}}$ при $0<u<1$ являются очень быстро убьвающими функциями $m$. Так как при этом согласно неравенствам (2.15) величины $\gamma_{m}(u)$ и $\beta_{m}(u)$ являются монотонно возрастающими и ограниченными функциями $m$, то функции $M(z)$ и $N(z)$, определенные рядами $(2.16),(2.17)$, являются цельми трансцендентными функциями нулевого порядка.

Таким образом последовательности полиномов $M_{n}(z)$ и $N_{n}(z)$ сходятся к цельпм трансцендентным функциям.

Положим теперь $z=1$ и определим две функции

$$
\begin{aligned}
& f_{1}(x)=\sum_{m=0}^{\infty}(-1)^{m} u^{\pi_{m}} \gamma_{m}(u) x^{m}, \\
& f_{2}(x)=\sum_{m=0}^{\infty}(-1)^{m} u^{\sigma_{m}} \beta_{m}(u) x^{m} .
\end{aligned}
$$

Эти функции, очевидно, также являются целыми трансцендентными функциями нулевого порядка. Учитывая формулу (1.7), а также используя (2.16), (2.17), получаем следуюший результат.

ТЕорема 2.1. Функция Вейля $R(\lambda)$ рассматриваемого класса операторов представима в виде

$$
R(\lambda)=\lim _{n \rightarrow \infty} R_{n}(\lambda)=-\frac{f_{1}\left(\lambda^{-2}\right)}{\lambda f_{2}\left(\lambda^{-2}\right)},
$$

где $f_{1}(x)$ и $f_{2}(x)$ - челые функиии нулевого порядка, имеющие представление рядами Тейлора (2.19), (2.20).

Собственные значения оператора соответствуют полюсам функции $R(\lambda)$, которые определяются, в свою очередь, корнями уравнения $f_{2}\left(\lambda^{-2}\right)=0$. Так как собственные значения оператора $A$ образуют бесконечное множество с точкой сгущения $\lambda=0$, то функция $f_{2}(x)$ (а следовательно, и функция $f_{1}(x)$ ) имеет бесконечное число нулей (значение нуль не является особой точкой Пикарацелой функции). Непосредственньм следствием вещественности и простоты собственных значений оператора $A$ является следующее утверждение относительно нулей функций $f_{1}(x)$ и $f_{2}(x)$.

СлЕДСТВИЕ. Нули функиий $f_{1}(x)$ u $f_{2}(x)$ являются простыми, вещественными, положительными и перемежаются друг с другом.

Это следствие, являющееся практически очевидным с точки зрения спектральной теории и формулы (2.21), является отнюдь не тривиальным, если исходить из представления функций $f_{1}(x)$ и $f_{2}(x)$ рядами Тейлора $(2.19),(2.20)$.

Из формулы (2.21) следует, что спектр оператора $A$ расположен симметрично относительно предельной точки $\lambda=0$, являющейся существенно особой точкой функции Вейля $R(\lambda)$. Обозначим корни функции $f_{2}(x)$ через $x_{n}\left(f_{2}\left(x_{n}\right)=0\right)$, а собственные значения через $\pm \lambda_{n}\left(\lambda_{n}>0\right)$. Тогда

$$
\lambda_{n}=\frac{1}{\sqrt{x_{n}}} .
$$


Функция $R(\lambda)$ является матричньм элементом резольвенты оператора $A$. Для нее должно иметь место резольвентное разложение

$$
R(\lambda)=\sum_{n} \frac{R_{n}^{( \pm)}}{ \pm \lambda_{n}-\lambda}
$$

Группируя слагаемые с противоположными знаками $\lambda_{n}$, можем записать это равенство в виде

$$
R(\lambda)=\sum_{n} \frac{\lambda_{n}\left(R_{n}^{(-)}-R_{n}^{(+)}\right)-\lambda\left(R_{n}^{(-)}+R_{n}^{(+)}\right)}{\lambda^{2}-\lambda_{n}^{2}} .
$$

Здесь суммирование производится по абсолютным величинам собственных значений $\lambda_{n}$. Сравнивая это выражение с формулой (2.21), получаем равенство

$$
\frac{f_{1}\left(\lambda^{-2}\right)}{f_{2}\left(\lambda^{-2}\right)}=-\lambda \sum_{n} \frac{\lambda_{n}\left(R_{n}^{(-)}-R_{n}^{(+)}\right)-\lambda\left(R_{n}^{(-)}+R_{n}^{(+)}\right)}{\lambda^{2}-\lambda_{n}^{2}} .
$$

Но левая часть этого равенства является функцией $\lambda^{2}$. Чтобы и правая часть была функцией $\lambda^{2}$ необходимо, чтобы $R_{n}^{(+)}=R_{n}^{(-)}=R_{n}$, и мы получаем, таким образом,

$$
\frac{f_{1}\left(\lambda^{-2}\right)}{f_{2}\left(\lambda^{-2}\right)}=2 \lambda^{2} \sum_{n} \frac{R_{n}}{\lambda^{2}-\lambda_{n}^{2}}
$$

или, учитывая (2.22),

$$
\frac{f_{1}(x)}{f_{2}(x)}=2 \sum_{n} \frac{x_{n} R_{n}}{x_{n}-x}
$$

3. Асимптотика собственных значений. Скорость стремления к нулю абсолютных величин собственных значений $\lambda_{n}$ определяется согласно $(2.22)$ скоростью роста корней $x_{n}$ функции $f_{2}(x)$. Поэтому достаточно найти асимптотику $x_{n}$. Функция $f_{2}(x)$ определялась рядом (2.20):

$$
f_{2}(x)=\sum_{m=0}^{\infty}(-1)^{m} u^{\sigma_{m}} \beta_{m}(u) x^{m}=\sum_{m=0}^{\infty} a_{m} x^{m}=\sum_{m=0}^{\infty} v_{m}(x) .
$$

Согласно теореме 2.1 эта функция является целой трансцендентной функцией нулевого порядка. Если $N(r)$ - число нулей $f_{2}(x)$, не превосходящих $r$, то согласно известной теореме [5] порядок функции $N(r)$ также равен нулю. То есть функция $N(r)$ возрастает медленнее любого полинома от $r$. Грубо можно оценить асимтотику $x_{n}$ как

$$
x_{n} \sim \frac{1}{\left(a_{n}\right)^{1 / n}}, \quad n \rightarrow \infty,
$$

откуда, учитьвая формулы (2.15) и (2.18), грубо имеем

$$
x_{n} \sim\left(\frac{1}{u}\right)^{\frac{(2 n)^{s}}{s+1}}=\left(\frac{1}{q}\right)^{\frac{2(2 n)^{s}}{s+1}} .
$$


Перейдем к более точньм оценкам. Найдем максимальньй по модулю член в ряде (3.1). Приравнивая логарифмическую производную от $\left|v_{n}\right|$ к нулю и учитывая (2.15), (2.18), найдем, что асимптотически при больших $r=|x|$ номер максимального коэффициента равен

$$
n_{0}=\frac{1}{2}\left(\frac{\ln r}{\ln u^{-1}}\right)^{1 / s} \cdot\left(1+O\left(n_{0}^{-2}\right)\right) \text {. }
$$

Возьмем достаточно большое $n_{0}$ и положим

$$
r_{0}=u^{-\left(2 n_{0}\right)^{s}}, \quad n_{0}=\frac{1}{2}\left(\frac{\ln r_{0}}{\ln u^{-1}}\right)^{1 / s}
$$

Далее, учитьвая определение величины $\sigma_{n}$, имеем

$$
\left|\frac{v_{n+1}(x)}{v_{n}(x)}\right|=r u^{(2 n+1)^{s}} \frac{\beta_{n+1}(u)}{\beta_{n}(u)}
$$

Полагая $r=r_{0}$, получим неравенства

$$
\begin{aligned}
\left|v_{n_{0}+1}\right| & =u^{\left(2 n_{0}+1\right)^{s}-\left(2 n_{0}\right)^{s}} \frac{\beta_{n_{0}+1}(u)}{\beta_{n_{0}}(u)}\left|v_{n_{0}}\right| \leqslant u^{s\left(2 n_{0}\right)^{s-1}} \frac{\beta_{n_{0}+1}(u)}{\beta_{n_{0}}(u)}\left|v_{n_{0}}\right|, \\
\left|v_{n_{0}+2}\right| & =u^{\left(2 n_{0}+3\right)^{s}-\left(2 n_{0}\right)^{s}} \frac{\beta_{n_{0}+2}(u)}{\beta_{n_{0}+1}(u)}\left|v_{n_{0}+1}\right| \leqslant u^{3 s\left(2 n_{0}\right)^{s-1}} \frac{\beta_{n_{0}+2}(u)}{\beta_{n_{0}+1}(u)}\left|v_{n_{0}+1}\right| \\
& \leqslant u^{4 s\left(2 n_{0}\right)^{s-1}} \frac{\beta_{n_{0}+2}(u)}{\beta_{n_{0}}(u)}\left|v_{n_{0}}\right|, \\
\left|v_{n_{0}+3}\right| & \leqslant u^{9 s\left(2 n_{0}\right)^{s-1}} \frac{\beta_{n_{0}+3}(u)}{\beta_{n_{0}}(u)}\left|v_{n_{0}}\right| \quad \text { и т.д. }
\end{aligned}
$$

Поэтому

$$
\left|\sum_{m=n_{0}+1}^{\infty} v_{m}\left(x_{0}\right)\right| \leqslant C\left|v_{n_{0}}\right| \sum_{m=1}^{\infty} u^{m^{2} s\left(2 n_{0}\right)^{s-1}} .
$$

Здесь мы использовали неравенства (2.15).

Аналогично при $r=r_{0}$ имеем также следуюшие неравенства:

$$
\begin{aligned}
\left|v_{n_{0}-1}\right| & =u^{\left(2 n_{0}\right)^{s}-\left(2 n_{0}-1\right)^{s}} \frac{\beta_{n_{0}-1}(u)}{\beta_{n_{0}}(u)}\left|v_{n_{0}}\right|<u^{s\left(2 n_{0}-1\right)^{s-1}} \frac{\beta_{n_{0}-1}(u)}{\beta_{n_{0}}(u)}\left|v_{n_{0}}\right| \\
\left|v_{n_{0}-2}\right| & =u^{\left(2 n_{0}\right)^{s}-\left(2 n_{0}-3\right)^{s}} \frac{\beta_{n_{0}-2}(u)}{\beta_{n_{0}-1}(u)}\left|v_{n_{0}-1}\right| \\
& <u^{3 s\left(2 n_{0}-3\right)^{s-1}+s\left(2 n_{0}-1\right)^{s-1}} \frac{\beta_{n_{0}-2}(u)}{\beta_{n_{0}}(u)}\left|v_{n_{0}}\right| \quad \text { и т.д. }
\end{aligned}
$$

И, следовательно,

$$
\left|\sum_{m=0}^{n_{0}-1} v_{m}\left(x_{0}\right)\right| \leqslant C\left|v_{n_{0}}\right| \sum_{m=1}^{n_{0}} u^{\left(s\left(2\left(n_{0}-1\right)+1\right)^{s-1}+\cdots+(2 m-1) s\left(2\left(n_{0}-m\right)+1\right)^{s-1}\right)} .
$$


Из формул (3.3), (3.4) следует, что при больших по модулю $x_{0}=r_{0} e^{i \varphi}$ и при $s \neq 1$

$$
f_{2}\left(x_{0}\right)=v_{n_{0}}\left(x_{0}\right)\left(1+\epsilon\left(x_{0}\right)\right)=(-1)^{n_{0}} u^{\sigma_{n_{0}}-2^{s} n_{0}^{s+1}} \beta_{n_{0}}(u) e^{i n_{0} \varphi}\left(1+\epsilon\left(x_{0}\right)\right),
$$

причем

$$
\lim _{r_{0} \rightarrow \infty} \epsilon\left(x_{0}\right)=0, \quad 0 \leqslant \varphi \leqslant 2 \pi .
$$

Из этой формулы следует, что изменение аргумента функции $f_{2}\left(x_{0}\right)$ при обходе окружности достаточно большого радиуса $R=r_{0}$ в точности равно $2 \pi n_{0}$. Поэтому по теореме Коши получаем, что число корней $N$ функции $f_{2}$ в круге достаточно большого радиуса $r=u^{-(2 N)^{s}}$ с учетом (3.2) в точности равно

$$
N(r)=\frac{1}{2}\left(\frac{\ln r}{\ln u^{-1}}\right)^{1 / s}, \quad s \neq 1
$$

Метод, которьм мы здесь воспользовались для определения асимптотики корней, в сущности, аналогичен методу, которьй применялся Г. Харди [6] к функциям вида $\sum x^{\phi(n)} /\{\phi(n)\}$ !, где $\phi(n)$ - достаточно быстро растущая функция.

Рассмотрим теперь случай $s=1$. Если в предыдущем случае рост функции определялся максимальньм по модулю членом ряда и мы имели $\lim _{r_{0} \rightarrow \infty} \epsilon\left(x_{0}\right)=0$, то здесь уже $\epsilon\left(x_{0}\right)$ не стремится к нулю. Поэтому можно было бы воспользоваться и тем же самым методом, но результат был бы справедлив далеко не для всех значений $u$. Чтобы получить результат, справедливый при всех значениях $u$, воспользуемся методом, которьй также восходит к Харди [7].

При $s=1$ из предыдуших формул имеем

$$
\sigma_{m}=(2 m-1)+(2 m-3)+\cdots+3+1=m^{2}, \quad \beta_{m}(u)=\frac{1}{(1-u)\left(1-u^{2}\right) \cdot \ldots \cdot\left(1-u^{m}\right)}
$$

и, следовательно,

$$
f_{2}(x)=\sum_{m=0}^{\infty}(-1)^{m} \frac{u^{m^{2}}}{(1-u)\left(1-u^{2}\right) \cdot \ldots \cdot\left(1-u^{m}\right)} x^{m}=\sum_{m=0}^{\infty} v_{m}(x)
$$

Это известная функция Роджерса-Рамануджана [4].

Выбирая достаточно большое $N$ и полагая $M=[\ln N]$, разделим этот ряд на три части

$$
f_{2}(x)=\sum_{m=0}^{N-M-1} v_{m}(x)+\sum_{m=N-M}^{N+M} v_{m}(x)+\sum_{m=N+M+1}^{\infty} v_{m}(x)=A+B+D .
$$

Полагая $x=r e^{i \varphi}$ и $r=u^{-2 N}$, оценим каждую из частей:

$$
B=u^{-N^{2}} e^{i N(\varphi-\pi)} \beta(u) \sum_{m=-M}^{M} u^{m^{2}} e^{i m(\varphi-\pi)} \frac{\beta_{N+m}(u)}{\beta(u)}
$$


Taк как

$$
\frac{\beta_{N+m}(u)}{\beta(u)}=\prod_{k=N+m+1}^{\infty}\left(1-u^{k}\right)
$$

то

$$
B=u^{-N^{2}} e^{i N(\varphi-\pi)} \beta(u) \sum_{m=-M}^{M} u^{m^{2}} e^{i m(\varphi-\pi)}\left(1+\rho_{m}\right), \quad \rho_{m}=O\left(u^{N+m+1}\right) .
$$

Представим $B$ в виде

$$
B=B_{1}-B_{2}-B_{3}+B_{4}
$$

где

$$
\begin{aligned}
& B_{1}=u^{-N^{2}} e^{i N(\varphi-\pi)} \beta(u) \sum_{m=-\infty}^{\infty} u^{m^{2}} e^{i m(\varphi-\pi)}, \\
& B_{2}=u^{-N^{2}} e^{i N(\varphi-\pi)} \beta(u) \sum_{m=M+1}^{\infty} u^{m^{2}} e^{i m(\varphi-\pi)} \\
& B_{3}=u^{-N^{2}} e^{i N(\varphi-\pi)} \beta(u) \sum_{m=-M-1}^{\infty} u^{m^{2}} e^{i m(\varphi-\pi)} \\
& B_{4}=u^{-N^{2}} e^{i N(\varphi-\pi)} \beta(u) \sum_{m=-M}^{M} \rho_{m} u^{m^{2}} e^{i m(\varphi-\pi)}
\end{aligned}
$$

Так как

$$
\left|\sum_{m=M+1}^{\infty} u^{m^{2}} e^{i m(\varphi-\pi)}\right| \leqslant \sum_{M+1}^{\infty} u^{m^{2}}<C u^{M^{2}}, \quad\left|\sum_{m=-M-1}^{-\infty} u^{m^{2}} e^{i m(\varphi-\pi)}\right|<C u^{M^{2}}
$$

где $C$ - постоянная, учитьвая, что $M=[\ln N]$, имеем для $B_{2}$ и $B_{3}$ оценки

$$
\left|B_{2}\right|<C_{1} u^{\ln ^{2} N} u^{-N^{2}}, \quad\left|B_{3}\right|<C_{1} u^{\ln ^{2} N} u^{-N^{2}},
$$

где $C_{1}$ - также постоянная. Для $B_{4}$ получим следующую оценку:

$$
\left|B_{4}\right|<C u^{-N^{2}} \sum_{m=-M}^{M} u^{N+m+1}<C u^{N-\ln N} u^{-N^{2}}
$$

Обозначим

$$
\eta(\varphi)=\sum_{m=-\infty}^{\infty} u^{m^{2}} e^{i m(\varphi-\pi)}
$$

Эта функция непосредственно связана с эллиптической функцией $\vartheta_{3}(v)[8]$ :

$$
\eta(\varphi)=\vartheta_{3}(v)=\sum_{m=-\infty}^{\infty} u^{m^{2}} e^{2 \pi i m v}, \quad v=\frac{\varphi-\pi}{2 \pi}
$$


$\Phi$ ункция $\vartheta_{3}(v)$ раскладывается в бесконечное произведение вида [7]

$$
\vartheta_{3}(v)=\prod_{k=1}^{\infty}\left(1-u^{2 k}\right) \prod_{k=1}^{\infty}\left(1+u^{2 k-1} e^{2 \pi i v}\right)\left(1+u^{2 k-1} e^{-2 \pi i v}\right) .
$$

Из (3.9) непосредственно следует, что все корни функции $\eta(\varphi)$ чисто мнимые, так как $0<u<1$. Поэтому

$$
|\eta(\varphi)|>C>0, \quad 0 \leqslant \varphi \leqslant 2 \pi .
$$

Объединяя полученные результаты и учитьвая (3.6)-(3.8) и $(3.10)$, получим

$$
B=B_{1}(1+\epsilon)=u^{-N^{2}} e^{i N(\varphi-\pi)} \eta(\varphi) \beta(u)(1+\epsilon), \quad|\epsilon|<C u^{\ln ^{2} N} .
$$

Перейдем теперь к оценке величин $A$ и $D$. Мы имеем следуюшие неравенства при $r=u^{-2 N}$ :

$$
|A| \leqslant \sum_{m=0}^{N-M-1}\left|v_{m}\right|<(N-M)\left|v_{N-M}\right|
$$

и

$$
|D| \leqslant \sum_{m=N+M+1}^{\infty}\left|v_{m}\right|<\left|v_{N+M}\right|\left(1+\frac{\left|v_{N+M+1}\right|}{\left|v_{N+M}\right|}+\frac{\left|v_{N+M+1}\right|}{\left|v_{N+M}\right|} \frac{\left|v_{N+M+2}\right|}{\left|v_{N+M+1}\right|}+\cdots\right) .
$$

Но при $n \geqslant N+M$ справедливо неравенство

$$
\frac{\left|v_{n+1}\right|}{\left|v_{n}\right|}=u^{2(n-N)+1} \frac{\beta_{n+1}}{\beta_{n}}<C u^{2 \ln N} .
$$

Поэтому

$$
|D|<C\left|v_{N+M}\right| \text {. }
$$

Для величин $\left|v_{N \pm M}\right|$ нетрудно получить следующую оценку:

$$
\left|v_{N \pm M}\right|<C u^{-N^{2}} u^{M^{2}}=C u^{-N^{2}} u^{\ln ^{2} N},
$$

и для величин $A$ и $D$ окончательно имеем

$$
|A|<C u^{-N^{2}} N u^{\ln ^{2} N}, \quad|D|<C u^{-N^{2}} u^{\ln ^{2} N} .
$$

Из (3.11) и (3.12) найдем асимптотику функции $f_{2}(x)$

$$
f_{2}(x)=u^{-N^{2}} e^{i N(\varphi-\pi)} \eta(\varphi) \beta(u)(1+\epsilon), \quad N=\frac{\ln r}{2 \ln \left(u^{-1}\right)}, \quad \epsilon=O\left(N u^{\ln ^{2} N}\right) .
$$

Из формулы (3.13) сразу следует, что число корней функции $f_{2}$ внутри окружности достаточно большого радиуса $r=u^{-2 N}$ в точности равно

$$
N(r)=\frac{\ln r}{2 \ln \left(u^{-1}\right)},
$$

так как изменение аргумента функции $\eta(\varphi)$ при обходе этой окружности в силу (3.9) равно нулю.

Таким образом, мы доказали, что формула (3.5) справедлива для любого натурального $s$. Отсюда, учитывая, что корни являются вещественньпи и положительными, получим асимптотическую оценку нулей функции $f_{2}$ :

$$
u^{-(2 n-2)^{s}}<x_{n}<u^{-(2 n)^{s}}, \quad n>N, \quad s=1,2, \ldots .
$$

Учитывая формулу (2.22), получаем следующую теорему. 
ТЕОрема 3.1. Для абсолютных величин собственных значений $\lambda_{n} \quad(n=0,1, \ldots)$ оператора А справедлива следующая асимптотическая оценка:

$$
b_{2 n+1}<\lambda_{n}<b_{2 n-1}, \quad n>N \text {, }
$$

әде $N$ - достаточно большое челое число.

В заключение докажем следующее простое предложение.

Теорема 3.2. Если $q>1$, то оператор А неограничен и имеет индексы дефекта $(1,1)$, т.е. проблема моментов полностью не определена.

ДокАЗАТЕльСТво. Если $q>1$, то мы имеем следующие условия:

$$
\begin{aligned}
\sum_{n=0}^{\infty} \frac{1}{b_{n}}<\infty, & \\
b_{n-1} b_{n+1} \leqslant b_{n}^{2}, & 0<s \leqslant 1, \\
b_{n-1} b_{n+1}>b_{n}^{2}, & s>1 .
\end{aligned}
$$

Таким образом, вьполняются все условия теоремы, являющейся аналогом одной теоремы Ю. М. Березанского [3] и, следовательно, оператор $A$ является симметрическим с индексами дефекта $(1,1)$. Условие $(* *)$ в [3] отсутствует, однако его можно рассматривать наравне с условием $(*)$ (доказательство остается тем же).

\section{СПИСОК ЦИТИРОВАННОЙ ЛИТЕРАТУРЫ}

[1] Ахиезер Н., Крейн М. О некоторых вопросах теории моментов. М.: ГОНТИ, 1938.

[2] Stone M. Linear Transformations in Hilbert Space and Their Applications to Analysis. Providence, RI: American Mathematical Society, 1932.

[3] Ахиезер Н. И. Классическая проблема моментов. М., 1961.

[4] Эндрюс Г. Теория разбиений. М.: Мир, 1982.

[5] Левин Б. Я. Распределение корней целњх функций. М., 1956.

[6] Hardy G. On the zeroes of certain classes of integral Taylor series. Part $1 / /$ Collected Papers of G. Hardy. V. 4, 1969. P. 54.

[7] Hardy G. The zeros of the integral function $\sum x^{n^{2}} /\left(n^{2}\right)$ !, and some similar functions // Collected Papers of G. Hardy. 1969. V. 4. P. 118.

[8] Смирнов В. И. Курс высшей математики. Т. 3. Ч. 2. Л.-М., 1949.

E-mail: Teduard@cards.lanck.net

Поступило 20.05 .2001

Исправленный вариант

12.09.2002 